\title{
EXACTITUD DIAGNÓSTICA DE LA PRUEBA DE PROVOCACIÓN BRONQUIAL CON METACOLINA Y LA CLINICA DEL PACIENTE EN LA CONFIRMACIÓN DE ASMA EN NIÑOS
}

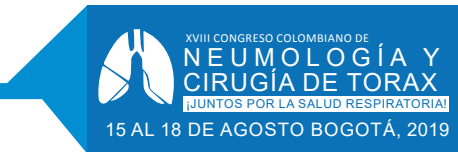

\section{Elida Dueñas, Sandra Mancera, Carlos Aguirre, Luis Fernando Giraldo}

\section{INTRODUCCIÓNY OBJETIVO}

El diagnóstico de asma es fundamentalmente clínico y las pruebas de función pulmonar lo apoyan; en un alto porcentaje de niños, la espirometría es normal y en ellos la prueba de provocación bronquial con metacolina puede establecer en conjunto con la clínica, la probabilidad de presentar la enfermedad. El objetivo de este estudio fue determinar la exactitud diagnóstica de la prueba de metacolina para confirmar el diagnóstico de asma en una cohorte de niños entre 6 y 15 años de edad.

\section{MATERIALES Y MÉTODOS}

Estudio de pruebas diagnósticas fase III, de corte transversal, en una cohorte de niños de 6 a 15 años con riesgo de tener asma a quienes se les practico la prueba de metacolina. Se compararon los valores de la prueba de metacolina frente al patrón de referencia clínico: criterios clínicos GINA 2017. La prueba de metacolina se realizó de acuerdo con ATS/ERS. Se construyeron curvas de rendimiento diagnóstico (curvas ROC), se calculó el área bajo la curva $(A U C-R O C)$ para $P C_{20}$, porcentaje de caída del $V_{E F}$ y respuesta al $B_{2}$ inhalado, se establecieron puntos de corte y se estimaron valores predictivos para el diagnostico

\section{RESULTADOS}

Se incluyerón 141 niños con riesgo de asma y prueba de metacolina, 116 de ellos con diagnóstico clínico de asma (severa: $18,4 \%$, moderada: $36,2 \%$, leve: $45.4 \%$ ). En la prueba de metacolina, 89 niños (63\%) tuvieron hiperreactividad bronquial: leve: $24,8 \%$; limitrofe: $22 \%$; moderada a severa: $16,3 \%$.

Los valores porcentuales promedio de la CVF, VEF 1 y VEF $/$ CVF fueron $109,5 \%, 100,1 \%$ y $83 \%$ (66-96\%) respectivamente. El porcentaje de respuesta del $V_{E F}$ al $B 2$ inhalado fue de $35,6 \%$. Las AUC-ROC para $P_{20}$, porcentaje de caída del VEF $F_{1} Y$ respuesta al $\mathrm{B}_{2}$ inhalado mostraron valores aceptables con significación estadística para el diagnóstico de asma: $P C_{20}$ (AUC-ROC: 0.7; IC 95\%: 0.59-0.82; P < 0.001), porcentaje de caida del VEF 1 (AUC-ROC: 0.77; IC 95\%: 0.66-0.87; P $<0.001$ ) y respuesta del VEF 1 al B2 (AUC-ROC: $0.7 ;$ IC 95\%: 0.61-0.84; $P<0.001$ ).

Probabilidad diagnóstica de asma

\begin{tabular}{|c|c|c|c|c|c|c|c|c|c|c|c|c|c|}
\hline \multicolumn{14}{|c|}{ Estodistios de Erotitud Diannóstia } \\
\hline & Crituso & \begin{tabular}{|l|} 
Senss \\
bilox:
\end{tabular} & ssod & \begin{tabular}{|l|} 
Espect \\
odsos
\end{tabular} & $955 \mathrm{al}$ & $+R$ & 9550 & $4 R$ & $95 \%$ & $+\mathrm{PV}$ & $958 \mathrm{a}$ & $\mathrm{PV}$ & $55 \mathrm{Ka}$ \\
\hline \multirow[t]{2}{*}{9020} & s1467 & 60.1 & $588 \cdot 76,4$ & 76 & $549 \cdot 906$ & 284 & $1,4 \cdot 5,8$ & 0,4 & $03 \cdot 0,6$ & $\$ 9$ & $86,6 \cdot 96,4$ & 339 & $267 \cdot 420$ \\
\hline & s16 & 70,69 & $615-788$ & 72 & 506.879 & 252 & 13.48 & 0,41 & $03 \cdot 0,6$ & $\$ 2,1$ & $86,1-5,5,7$ & 346 & $287 \cdot 435$ \\
\hline \multirow[t]{2}{*}{$\begin{array}{c}\text { scosist } \\
\text { V.5! }\end{array}$} & $5=10$ & 90,52 & 837.952 & 52 & $31,3 \cdot 722$ & 189 & $12 \cdot 2,8$ & 0,18 & $19 \cdot 0,4$ & 89,7 & $85,3 \cdot 93,0$ & 542 & $375 \cdot 69,9$ \\
\hline & 58 & 93,1 & 869.970 & 48 & $278 \cdot 68,7$ & 1,79 & $12 \cdot 26$ & 0,14 & $0,07 \cdot 0,3$ & 69.3 & $85,0 \cdot 924$ & 60. & $407 \cdot 76,7$ \\
\hline \multirow[t]{2}{*}{ Eversibildad } & $>11$ & 9052 & 837.952 & 4 & $24+65,1$ & 1,62 & $1,1 \cdot 23$ & 0,2 & $0,1 \cdot 04$ & 82 & $84,1 \cdot 91,4$ & \$N & 328.672 \\
\hline & $\$ 12$ & 87,93 & 806.932 & 4 & $244 \cdot 65,1$ & 1,57 & $1,1 \cdot 22$ & 027 & $0,1 \cdot 0,5$ & 879 & $83,6 \cdot 912$ & 4 & $289 \cdot 60.3$ \\
\hline
\end{tabular}

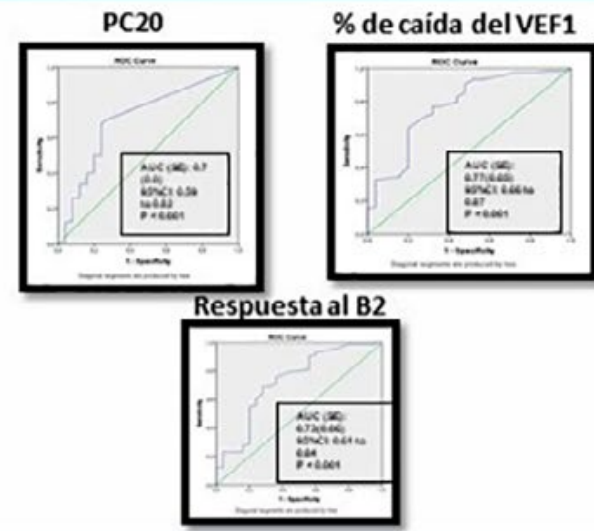

\section{CONCLUSIONES}

La prueba de metacolina tiene una capacidad diagnóstica aceptable para el diagnóstico de asma en niños; sin embargo, la caída del $\mathrm{VEF}_{1}$ tiene una mejor capacidad discriminatoria, respecto al comportamiento del $\mathrm{PC}_{20}$ y de la respuesta al broncodilatador inhalado.

Establecer un indice que reúna la probabilidad diagnostica clínica y la suma de los tres parámetros: respuesta del $\mathrm{PC}_{20}$. 